\title{
Iris Recognition Using Wavelet Transform and SVM BasedApproach
}

\author{
Sushilkumar S. Salve \\ Electronics and Telecommunication Department \\ Sinhgad College of Engineering \\ Pune, India \\ sushil.472@gmail.com
}

\begin{abstract}
Inthis paper we proposed an improved novel approach to identify the person using iris recognition technique. This approach is based on fuzzy and support vector machine (SVM) as an iris pattern classifier. Prior to classifier, region of interest i.e. iris region is segmented using Canny edge detector and Hough transform. Provided that the effect of eyelid and eyelashes get reduced. Daugman's rubber sheet model used to get normalized iris to improve computational efficiency and proper dimensionality. Further, discriminating feature sequence is obtained by feature extraction from segmented iris image using $1 D$ Log Gabor wavelet.Encoding is done using phase quantization to get feature vectors. These binary sequence feature vectors are used to train $S V M$ and fuzzy system as iris pattern classifier. The experimental tests are performed over standard CASIAIrisV4 database.
\end{abstract}

Keywords-iris, segmentation, Gabor filters, SVM,FCM

\section{INTRODUCTION}

Due to higher need of security in public as well as in personal information aspects, vigorousand reliable automatic recognition of individuals has been an attractive goal [1], [2], [5],[7],[8]. In public security and information security domain biometrics technology plays an important role. Biometrics is standard of comparison based on either physical or behavioral traits. Fingerprint, iris pattern are physical traits while voice, signature includes in behavioral traits. However, iris recognition is proven to be more beneficial as this accurately identifies each individual and also distinguishes one from another. The human iris is thin and annular region in eye located around pupil and covered by cornea, as shown in figure 1 , which able to provide unique and independent detail information of individual [3]. Random texture of protected iris is mostly stable throughout the life. Iris patterns are too unique even not only differ in between identical twins but also differ in between the right and left eye. Iris recognition requires comparing a register or enrolled iris template against a newly captured iris from eye of individual [9], [16]. The iris recognition is applicable in identification as well as in verification mode. In identification mode recognition system identifies a person from entire register template by searching a database for matching. Identification is 'one to many'matching style [18], [24], [29], [38]. In verification mode recognition system authenticate a person's claimed identity from their's previously enrolled patterns.

Verification is 'one to one' matching style.

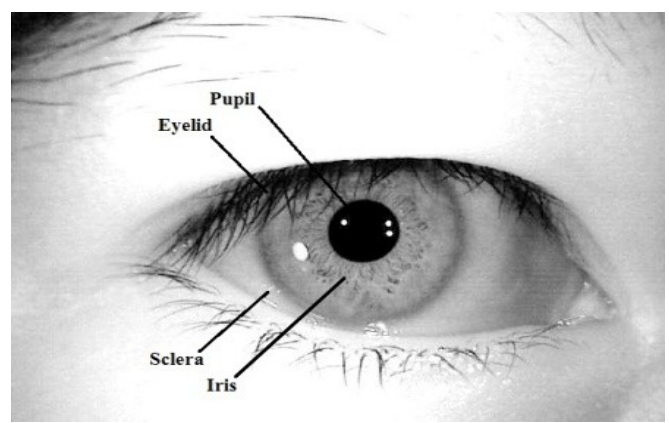

Figure 1.Human Eye

Here, our objective is to overcome the challenge like high recognition rate of recognition system.

\section{RELETED WORK}

Many researcher worked in the field of iris recognition. Daugman suggest first successful working iris recognition system [1]. Integro-differential operator uses first order derivatives of image intensity to locate circular edges of iris and upper and lower eyelids. By varying the radius $r$ and center $(\mathrm{x}, \mathrm{y})$ position of circular contour the operator searches for circular path for maximum change in pixel values. To achieve precise localization, the operator is applied iteratively with progressively reduced smoothing. In Daugman's system, by quantizing local phase angle of imaginary and real parts of 


\section{Asian Journal of Convergence in Technology ISSN NO: 2350-1146 I.F-5.11}

filtered image,feature vector of 2048b were generated [1], [3]. R. P. Wilde proposed a system which uses 4 level Laplacian to extract features of iris. After extracting features, normalized correlation and fisher classifier is used to classify the features [11].Tsai, Lin and Tao used fuzzy gray curve tracing method for isolate iris region. To extract local feature points and to evaluate dominant orientation of all detected feature points, multi-resolution Gabor filters are used. Fuzzy matching algorithm is also introduce to calculate matching score [3]. Wilde system compares feature point by using the information comprising the local feature and the position of each point [3]. K. Seetharaman and R. Raghupathy adopted Canny edge detection method and circular Hough transform to segment the iris [4]. Daugman's rubber sheet model used to convert polar coordinate to rectangular coordinate for fixed dimension iris [1], [4]. After decomposing 2D normalized iris to 1D signal, feature will be extracted by convolving that $1 \mathrm{D}$ signal with $1 \mathrm{D}$ Log-Gabor filter using FFT and IFFT. These feature given to phase quantization process as input, to generate binary template i.e. iris code. Hamming distance is used for bit-wise comparison for matching [1], [4]. Boles and Boashah wereanalyzed iris images by zero crossing of 1D wavelet transform. Zero crossing representation is used to extract features. Different dissimilarity functionsare used to compare feature vectors [10]. Ma, Tan extract local details of iris pattern were capture by bank of spatial filter which are kernel based on Gaussian--Hermite moment and key local variation method using 1D wavelet transform [28].Sun adopted Gaussian filter to calculate direction of iris image and then angle scale quantized into six discrete value. Yu uses multichannel Gabor filters to fetch key points of iris image. Related distances between these key points are used to represent features [3]. Hyun proposed score level based iris recognition method using SVM (Support Vector Machine) and two Gabor wavelet filters [25].

The remainder of this paper is organized as follows. Section III describes the proposed approach for iris segmentation. An approach for feature extraction andan encoding is described in Section IV. The proposed novel matching schemes by SVM and fuzzy system are presented in Section V and VI Sections respectively. The experimental results are shown in Section VII. At last, Section VIII concludes the research work.

\section{IRIS SEGMENTATION}

An eye image consist of iris texture and some irrelevant part as well [13], [15], [12], [17], [23]. So, to isolate region of interest i.e. annular iris region, the pupillary and limbic boundaries should be detected. In this section, we describe, proposed edge detection and inner and outer circle detection using Canny edge detector and Hough transform [26] respectively.

\section{A. Edge detection}

Every eye image is consist of different pixel values. Each pixel is associated with its illumination. So, the abrupt changes

\section{Volume V Issue I}

in illumination are used by edge detector to get edge map of image. Here we proposed multi-step edge detection method usingCanny edge detector. To apply Canny edge detection, basic image processing operation like smoothing, thresholding are used. The whole procedure is given below to apply Canny edge detection.

1. To reduce unwanted noise and texture Gaussian filter smooths the image with mathematical equation,

Where,

$$
\mathrm{g}(\mathrm{m}, \mathrm{n})=\mathrm{G}(\mathrm{m}, \mathrm{n}) * \mathrm{f}(\mathrm{m}, \mathrm{n})
$$

$$
G=\frac{1}{2 \sigma^{2}} e^{\left(\frac{-\left(m^{2}+n^{2}\right)}{2 \sigma^{2}}\right)}
$$

2. By using gradient operation, compute gradient of smoothed image $g(m, n)$. Mathematically, gradient of $\mathrm{g}(\mathrm{m}, \mathrm{n})$ computed as,

$$
M(m, n)=\sqrt{\left(g_{m}{ }^{2}(m, n)+g_{n}{ }^{2}(m, n)\right)}
$$

3. Thresholding

$$
M_{T}(m, n)=f(x)=\left\{\begin{array}{c}
M(m, n) \text { if } \underset{\dot{M}}{M}(m, n)>T \\
\text { ¿0otherwise }
\end{array}\right.
$$

4. Those non-zero $M_{T}(m, n)$ are greater than their two adjacent along direction of gradient keep them unchanged, otherwise set it to 0 .

5. Now set two threshold to get two binary images $T_{1}$ and $T_{2}$.

6. Then by linking edges in $T_{1}$ and $T_{2}$, we get final edge map.

The edge map of input eye image using canny edge detector is shown in figure 2(b).

\section{B. $\quad$ Iris and pupil boundary detection}

Now, the important task to find exact location of iris, it is also called iris localization. But it demands the radius and center co-ordinates of iris and pupil region. The standard computer vision algorithm, Hough transform plays an important role to determine parameter of pupil and iris. As iris and pupil are annular region, so we used circular Hough transform to get center co-ordinate and radius of iris and pupil so that, we get boundaries of respective regions.

By taking gradient of intensity of eye image and then thresholding the resultant image, we get an edge map of eye image. From an edge map, for the parameter of circle through an each edge point votes are cast in Hough space. $X_{c}$ and 


\section{Asian Journal of Convergence in Technology ISSN NO: 2350-1146 I.F-5.11}

$y_{c}$ are the center coordinates and radius $r$ are the parameter, by which we can define a circle according to equation,

$$
x_{c}+y_{c}-r^{2}=0
$$

Using linear Hough transform eyelid can be isolate by fitting line to upper and lower eyelids. The resultant boundary detection and suppression [14] of noisy eyelid, eyelashes are shown in figure 2(c) and figure 2(d) respectively.

\section{Normalization}

As we successfully segment the region of interest, i.e. isolated iris region. Now we need to have fixed dimension iris region so that we allow them for comparison. As illumination level gets vary the pupil dilation occurs. This causes stretching of iris and this is the reason for dimensionally inappropriate iris. The sources like camera rotation, imaging distance may cause the inconsistency in iris images. So to produce the iris region with fixed dimensions, we need normalization due to which we get two snaps of same iris in varying condition have the same important feature at same spatial location. Daugman suggested a rubber sheet model which re-assign each point of iris to polar coordinate $(r, \theta)$ where $r$ belong to interval $[0,1]$ and angle $\theta$ ranges in $[0,2 \longrightarrow \pi \quad]$. The remapping is mathematically modeled as, $I(x(r, \theta), y(r, \theta))_{\mathrm{I}}$

$$
r, \theta \quad)
$$

With $x(r, \theta)=(1-r) x_{p}(\theta)+r x_{i}(\theta)$

$$
y(r, \theta)=(1-r) y_{p}(\theta)+r y_{i}(\theta)
$$

$\mathrm{I}(\mathrm{x}, \mathrm{y})$ is iris image, $(\mathrm{x}, \mathrm{y})$ are original rectangular coordinates, $(r, \theta)$ are corresponding polar coordinates. The $x_{p}$ and $y_{p}$ are coordinates of pupil, $x_{i}$ and $y_{i}$ are coordinate of iris along angular directions $\theta$. This model compensate the inconsistencies due to imaging distance and pupil dilation and produces constant dimension normalized iris patterns. The normalized output image is as shown in figure 2(e).

\section{Volume V Issue I}

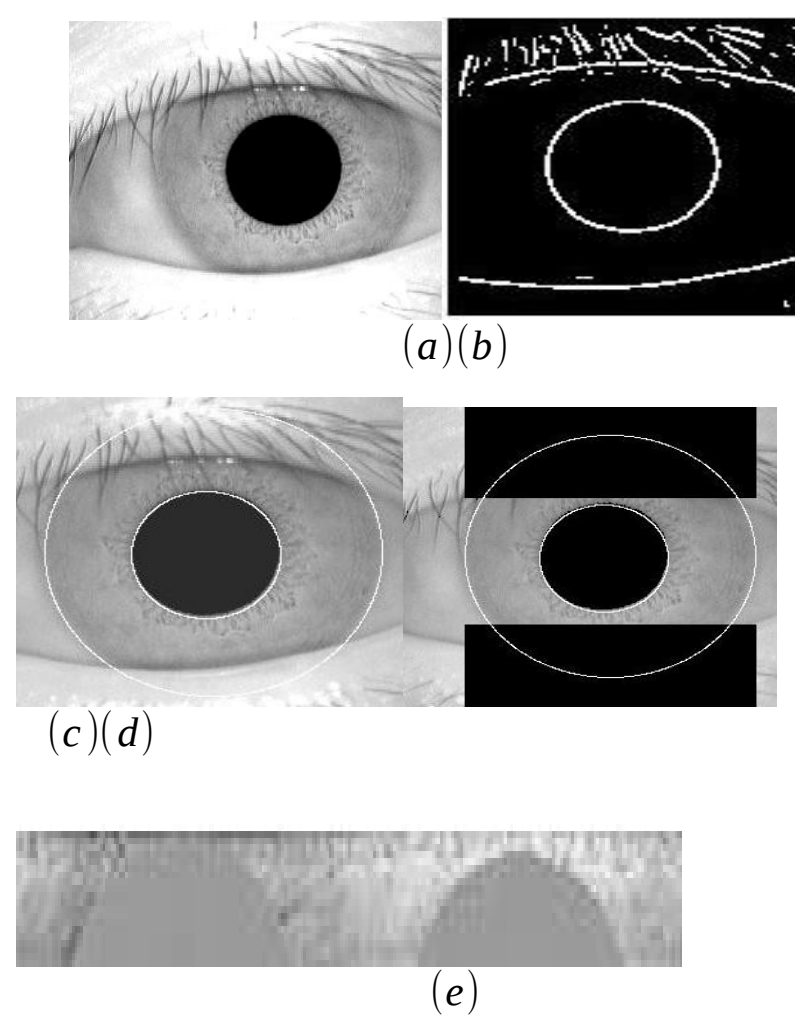

Figure 2.Segmentation Results

(a) Original eye image. (b) Edge map by canny edge detector. (c) After applying Hough transform. (d) Eyelid suppression. (e) Normalizes iris region.

\section{FEATURE EXTRACTION AND ENCODING}

To have accurate and precise recognition of person, the most useful and unique information about iris pattern must be extracted. For comparison purpose only significant information get encoded. Band pass decomposition [6], [17], [23], [19], [20], [22] of iris image is famous in iris recognition technique to create biometric template. In this paper, normalized iris pattern is convolved with 1D Log-Gabor wavelet for actual implementation of feature extraction. Number of 1D signal are recovered by breaking 2D normalized iris patterns. Then using FFT and IFFFT, convolution is done in between 1D signal and 1D Log-Gabor wavelet. Gabor wavelets are able to provide the best representation of signal in both space and spatial frequency. Sine/cosine wave get modulated with Gaussian to construct Gabor filter. Generally, a sine wave is much perfectly localizes in frequency than space. After sine modulated with Gaussian provide space localization but at same time it loses localization in frequency. The signal decomposition carried by Gabor filters. The drawback of Gabor filter is that, whenever the bandwidth exceeds one octave even symmetrical filter will have DC component. We can get the zero DC component using Gabor filter for any bandwidth which is nothing but Gaussian over logarithmic scale, which is known as Log- 


\section{Asian Journal of Convergence in Technology ISSN NO: 2350-1146 I.F-5.11}

Gabor wavelet [11], [23], [24], [25], [26], [27], [28] filter. Its frequency response is given as,

$$
G(f)=\exp \left(\frac{-\left(\log \left(\frac{f}{f_{0}}\right)\right)^{2}}{2\left(\log \left(\frac{\sigma}{f_{0}}\right)\right)^{2}}\right)
$$

Where, $f_{0}$ is center frequency, $\sigma$ is filter bandwidth.

The feature extraction is carried out by convolving 2D normalized iris with 1D Log Gabor wavelet. The 2D normalized iris is decomposed to $1 \mathrm{D}$ signal.1D signal is nothing but the row of 2D normalized iris pattern. Each row is corresponds to circular ring on iris region. Let us assume a 4

$\times 4$ matrix from normalized iris pattern,

$$
\left[\begin{array}{l}
120125150160 \\
120123156166 \\
125125155165 \\
124124154164
\end{array}\right]
$$

After convolution with 1D Log Gabor wavelet filter, the following complex number matrix is produced as feature.

$$
\left[\begin{array}{l}
-3.14-i 0.23-1.44-i 2.821 .75-i 2.693 .23+i 0.17 \\
-3.68-i 0.27-1.67-i 3.312 .06-i 3.143 .76+i 0.21 \\
-3.33-i 0.23-1.58-i 3.001 .86-i 2.923 .48+i 0.17 \\
-3.32-i 0.18-1.61-i 2.971 .82-i 2.923 .46+i 0.14
\end{array}\right.
$$

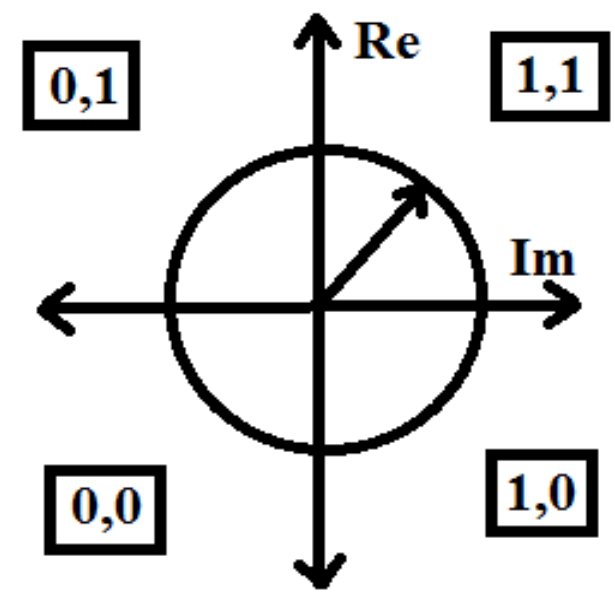

Volume V Issue I

Figure 3.Phase quantization

The feature extracted using 1D Log Gabor filter need to be encoded so that binary iris code will generate and it helps in classification in matching scheme. Phase quantization is used here to generate iris code. As represents in figure 3, phase quantization assigns 11 if both real and imaginary parts are positive, assigns 0,1 if real and imaginary parts are negative andpositive respectively, assigns 1,0 if real and imaginary parts are positive and negative respectively, and assigns 00 if both real and imaginary parts are negative. On the basis of this logic binary stream get generated as shown in matrix form,

$$
,\left[\begin{array}{l}
00001011 \\
00001011 \\
00001011 \\
00001011
\end{array}\right]
$$

This binary stream looks like as shown in figure 4,

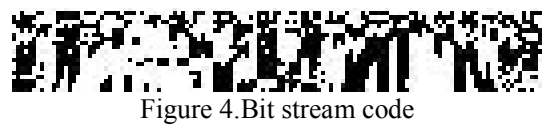

\section{MULTI CLASS SVM AS IRIS PATTERN CLASSIFIERS}

The code extracted from input iris image need to compare with previously enrolled iris code. This comparison is done based on Support vector machine (SVM) classifier [21], [31], [33], [34], [36], [39]. By finding decision surface between two classes of input vectors from training set. As shown in figure 5, SVM tries to maximize the distance of separating boundary of two classes by maximizing distance of separating plane [32] of each of feature vector.

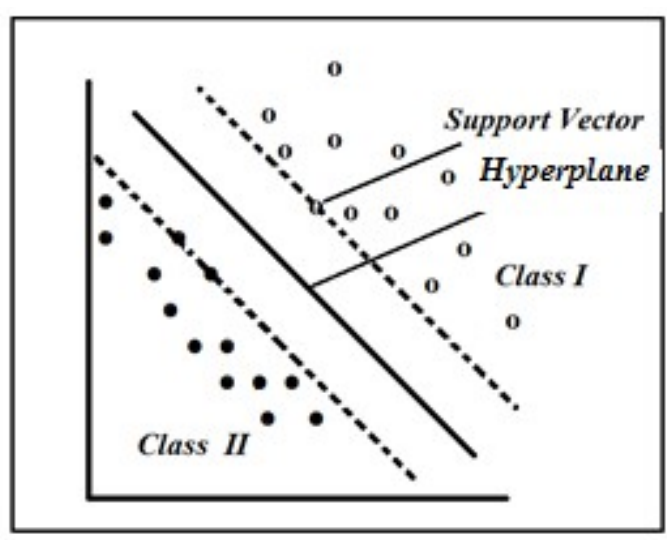

Figure 5: SVM classifier

If $\mathrm{x}_{\mathrm{i}}$ is input vector then $\mathrm{g}(\mathrm{x})$ is linear function given as,

$$
g(x)=w \cdot x_{i}+b
$$




\section{Asian Journal of Convergence in Technology ISSN NO: 2350-1146 I.F-5.11}

$\mathrm{g}(\mathrm{x})$ represents straight line if $\mathrm{x}$ is two dimensional feature vector. If it is 3 dimensional then $\mathrm{g}(\mathrm{x})$ represents plane. Now, $\mathrm{x}$ is greater than 3 dimension then $\mathrm{g}(\mathrm{x})$ represents hyper-plane as shown in figure 5. Here, $w$ is weight term which is perpendicular to hyper-plane. Orientation of hyper-plane is given by w. Also, b is bias term which indicates position of hyper-plane. Hyper-plane divides D dimensional space into two half spaces. In two class problem classification rule if

$$
\begin{aligned}
& g(x)=w . x_{i}+b>0 \quad \text { then, } \mathrm{x}_{\mathrm{i}} \text { belong to class } \mathrm{c}_{1} \text {. If } \\
& g(x)=w . x_{i}+b<0 \quad \text { then, } \mathrm{x}_{\mathrm{i}} \text { belongs to class } \mathrm{c}_{2} \text {. While }
\end{aligned}
$$

classifying any input vector we assign class belongingness $\mathrm{y}_{\mathrm{i}}=$

$$
\pm 1 \text {.If } x_{i} \in C_{1} \text { then } y_{i}= \pm 1 \text { and if } x_{i} \in C_{2}
$$

then $y_{i}=-1$. Hence $y_{i}\left(w \cdot x_{i}+b\right)>0 \quad$ is always positive whether $X_{i}$ belongs to $C_{1}$ or $C_{2}$. Now for stability of each feature vector we consider a margin $(y)$.Therefore, $w . x_{i}+b>\gamma$. This margin is nothing but a measure of distance of $X_{i}$ from hyper-plane. So, distance of hyperplane from that point $\quad x_{i}$ is given by $\frac{w \cdot x+b}{\|w\|} \geq \gamma$. By proper scaling we can set $\gamma \cdot\|w\|=1$. Therefore, $\mathrm{w} . \mathrm{x}+\mathrm{b}=1$. Now classification rule becomes,

$$
w . x_{i}+b \geq 1 \rightarrow x_{i} \epsilon c_{1} \wedge w . x_{i}+b \leq 1 \rightarrow x_{i} \epsilon c_{2}
$$

If $x_{i}$ is support vector then $y_{i}$ (w. $\left.x_{i}+\mathrm{b}=1\right)$.To design SVM we need margin as large as possible. So, margin will be $\frac{w \cdot x+b}{\|w\|} \geq \gamma$. To get largest possible margin two point take into consideration. (1) Minimize $\|w\|$

maximize $b$. This problem can be convert to un-constraint optimization problem by using Lagrangian multiplier.

$$
L(w, b, \alpha)=\frac{1}{2}\|w\|^{2}-\sum_{i=1}^{N} \alpha_{i}\left(y_{i}\left(x_{i} \cdot w+b\right)-1\right)
$$

Where, $\quad \alpha_{i}$ is Lagrangian multiplier. To optimize that take partial derivatives of above Lagrangian with respect to $\mathrm{w}$ and b. So we get,

$$
\frac{\partial L}{\partial b}=\sum_{i=1}^{m} \alpha_{i} y_{i}+0 \quad \text { And } \quad \frac{\partial L}{\partial b}=w-\sum_{i=1}^{m} \alpha_{i} y_{i}=0
$$

\section{Volume V Issue I}

Finally, we get

$$
L=\sum_{i=1}^{m} \alpha_{i}-\frac{1}{2} \sum \alpha_{i} \alpha_{j} y_{i} y_{j}\left(x_{i} \cdot x_{j}\right)
$$

To, become SV $\alpha_{i}$ must be very large. It means $\alpha_{i}$ shows the effect of feature vector on decision boundary. In order to obtain a non-linear decision boundary, we replace the inner product $\left(x_{i}, x_{j}\right)$ with a nonlinear kernel

$$
\begin{aligned}
& K\left(x_{i} \cdot x_{j}\right) \wedge g e t \\
& f(x)=\sum_{i=1}^{N} y_{i} \alpha_{i} K\left(x . x_{i}\right)+b
\end{aligned}
$$

The simple idea behind the nonlinear SVM [9] is to use a kernel function $K\left(x, x_{i}\right)$ to map the data $\mathrm{x}$ from the input space to the new higher dimensional feature space on which the mapped data points become linearly separable. The hidden units on the hidden layer are characterized by the kernel function adopted by SVM. The two basic kernels used in this work are,

Radial Basis Function (RBF) kernel:

$$
K\left(x, x_{i}\right)=\exp \left\{\frac{-\left\|x-x_{i}\right\|^{2}}{2 \sigma^{2}}\right\}
$$

Polynomial kernel: $\quad K\left(x, x_{i}\right)=\left(1+\frac{x \cdot x_{i}}{\sigma^{2}}\right)^{2}$

\section{FUZZY BASED IRIS PATTERN CLASSIFICATION}

Fuzzy classification is process of grouping an individual having same characteristics into fuzzy set. We use Fuzzy CMeans (FCM) algorithm [3] for fuzzy classification of iris patterns.Fuzzy C-means is a method of clustering which allows one data point to belong to one or more clusters. Fuzzy partitioning is carried out through an iterative optimization of the objective function shown below, with the update of membership $u_{i j}$ and the cluster centers $C_{j}$ by,

$$
u_{i j}=\frac{1}{\sum_{k=1}^{c}\left(\frac{\left\|x_{i}-c_{j}\right\|}{\left\|x_{i}-c_{k}\right\|}\right)^{\frac{2}{m-1}}}
$$


Asian Journal of Convergence in Technology

ISSN NO: 2350-1146 I.F-5.11

$$
u_{i j}=\frac{1}{\left(\frac{\left\|x_{i}-c_{j}\right\|}{\left\|x_{i}-c_{1}\right\|}\right)^{\frac{2}{m-1}}+\ldots+\left(\frac{\left\|x_{i}-c_{j}\right\|}{\left\|x_{i}-k\right\|}\right)^{\frac{2}{m-1}}}
$$

Where, $\quad\left\|x_{i}-c_{j}\right\|$ is distance from point $i$ to current cluster's center $j, \quad\left\|x_{i}-c_{k}\right\| \quad$ is the distance from point $i$ to other cluster centers $k$.

$$
c_{j}=\frac{\sum_{i=1}^{N} u_{i j}^{m} \cdot x_{i}}{\sum_{i=1}^{N} u_{i j}^{m}}
$$

The iteration will stop when,

$$
\max _{i j}\left(\left|u_{i j}^{(k+1)}-u_{i j}^{(k)}\right|\right\}<\varepsilon,
$$

Where $\varepsilon$ is a termination criterion between 0 and 1 , whereas $k$ the iteration steps. This procedure converges to a local minimum or a saddle point $J_{m}$.

The algorithm is composed of the following steps:

1. Randomly select cluster center.

2. Initialize $U=\left[u_{i j}\right]$ matrix, $U^{(0)}$

Calculate the $u_{i j}$ using:

$$
u_{i j}=\frac{1}{\sum_{k=1}^{c}\left(\frac{\left\|x_{i}-c_{j}\right\|}{\left\|x_{i}-c_{k}\right\|}\right)^{\frac{2}{m-1}}}
$$

3. At k-step: calculate the centers vectors $C(k)=\left[c_{j}\right]$ with $U(k)$

\section{Volume V Issue I}

$$
c_{j}=\frac{\sum_{i=1}^{N} u_{i j}^{m} \cdot x_{i}}{\sum_{i=1}^{N} u_{i j}^{m}}
$$

4. Update $U^{k}, U^{k+1}$

$$
u_{i j}=\frac{1}{\sum_{k=1}^{c}\left(\frac{\left\|x_{i}-c_{j}\right\|}{\left\|x_{i}-c_{k}\right\|}\right) \frac{2}{m-1}}
$$

5. If $\left\|U^{(k+1)}-U^{(k)}\right\|<\varepsilon \quad \|$ or the minimum $\mathrm{J}$ is achieved, then STOP; otherwise return to step 2.

After successful fuzzy clustering we need to find out the distance between cluster center and feature points are determined by calculating Euclidean distance, which is given by,

$$
d_{E}(y, p)=\sqrt{\sum_{i=1}^{L}\left(y_{i}-p_{i}\right)^{2}}
$$

Where, $\mathrm{L}$ is the dimension of feature vector, $\quad y_{i}$ the $i^{\text {th }}$ component of unknown feature vector and $p_{i}$ is component of center of cluster.

\section{EXPERIMENTAL RESULTS}

In order to evaluate performance of SVM kernels and FCM we use freely available CASIA database which most widely used for iris biometric purposes. It was not possible to use all of the eye images from each database, since perfect segmentation success rates were not attained. Instead a sub-set of each database was selected, which contained only those images that were segmented successfully. 
Asian Journal of Convergence in Technology

ISSN NO: 2350-1146 I.F-5.11

\begin{tabular}{|c|c|}
\hline CASIA V4- Iris Interval & 200 \\
\hline CASIA V4- Iris Lamp & 200 \\
\hline CASIA V4- Iris Syn & 200 \\
\hline CASIA V4- Iris Thousand & 200 \\
\hline CASIA V4- Iris Twins & 200 \\
\hline Total & 1000 \\
\hline
\end{tabular}

\section{Segmentation Results}

While implementing segmentation technique, we use circular Hough transform to localize iris boundary and linear Hough transform to detect eyelids. Thresholding is used in order to suppress eyelashes. Here, onwards we show segmentation results on each dataset. While implementing the segmentation technique we take 1000 images of above five datasets containing 200 images of each. So the properly or correctly segmented iris images are as shown in Figure 6.

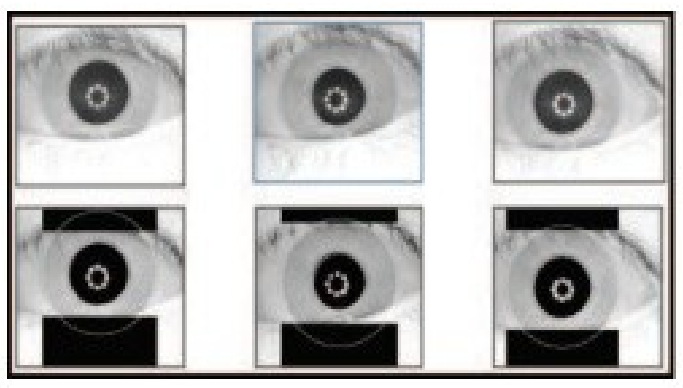

Figure 6: Segmentation result on CASIA V4 Iris Interval The overall segmentation results are summarized as follow,

TABLE II

COMPARISON OF VARIOUS METHODS USED FOR ESTIMATION

\begin{tabular}{|c|c|c|c|}
\hline Data Set & $\begin{array}{c}\text { Total no. of } \\
\text { Images }\end{array}$ & $\begin{array}{c}\text { Correctly } \\
\text { Segmented } \\
\text { Images }\end{array}$ & $\begin{array}{c}\text { Segmentatio } \\
\text { n Accuracy } \\
(\%)\end{array}$ \\
\hline $\begin{array}{c}\text { CASIA V4- } \\
\text { Iris Interval }\end{array}$ & 200 & 200 & 100 \\
\hline $\begin{array}{c}\text { CASIA V4- } \\
\text { Iris Lamp }\end{array}$ & 200 & 194 & 97 \\
\hline $\begin{array}{c}\text { CASIA V4- } \\
\text { Iris Syn }\end{array}$ & 200 & 197 & 98.5 \\
\hline $\begin{array}{c}\text { CASIA V4- } \\
\text { Iris Thousand }\end{array}$ & 200 & 185 & 92.5 \\
\hline $\begin{array}{c}\text { CASIA V4- } \\
\text { Iris Twins }\end{array}$ & 200 & 183 & 91.5 \\
\hline Total & 1000 & 959 & 95.9 \\
\hline
\end{tabular}

\section{Classification Results}

In this research work we use Support vector machine and FCM. In training phase we pass all 1000 images and take respective segmentation result and features are stored in the form of template.
Volume V Issue I

TABLE III

CLASSIFIXATION RESULTS USING FCM

\begin{tabular}{|c|c|c|c|}
\hline & $\begin{array}{c}\text { Total No. } \\
\text { of Images } \\
\text { (A) }\end{array}$ & $\begin{array}{l}\text { Correctly } \\
\text { Classified } \\
\text { Image } \\
\text { (B) }\end{array}$ & $\begin{array}{l}\text { Accuracy } \\
\text { B/A*100 }\end{array}$ \\
\hline $\begin{array}{l}\text { CASIA V4- Iris } \\
\text { Interval }\end{array}$ & 200 & 200 & 100 \\
\hline $\begin{array}{l}\text { CASIA V4- Iris } \\
\text { Lamp }\end{array}$ & 200 & 197 & 98.5 \\
\hline $\begin{array}{c}\text { CASIA V4- Iris } \\
\text { Syn }\end{array}$ & 200 & 194 & 96 \\
\hline $\begin{array}{c}\text { CASIA V4- Iris } \\
\text { Thousand }\end{array}$ & 200 & 188 & 94 \\
\hline $\begin{array}{l}\text { CASIA V4- Iris } \\
\text { Twins }\end{array}$ & 200 & 180 & 90 \\
\hline Total & 1000 & 959 & 95.9 \\
\hline
\end{tabular}

Radial basis function kernel and polynomial kernel are two kernels of SVM which we are implemented. The classification results given by using RBF kernel and polynomial kernel compared with FCM is as shown follow,

TABLE IV

ClassifiXATION RESUlTS USING SVM KERNELS

\begin{tabular}{|c|c|c|c|c|c|}
\hline & \multirow{2}{*}{$\begin{array}{l}\text { Total } \\
\text { No. of } \\
\text { Images } \\
\text { (A) }\end{array}$} & \multicolumn{2}{|c|}{$\begin{array}{l}\text { Correctly Classified } \\
\text { Images (B) }\end{array}$} & \multicolumn{2}{|c|}{$\begin{array}{l}\text { Accuracy } \\
\mathrm{B} / \mathrm{A} * 100\end{array}$} \\
\hline & & $\begin{array}{l}\text { Polynomial } \\
\text { Kernel }\end{array}$ & $\begin{array}{c}\text { RBF } \\
\text { Kernel }\end{array}$ & $\begin{array}{l}\text { Polynomial } \\
\text { Kernel }\end{array}$ & $\begin{array}{c}\text { RBF } \\
\text { Kernel }\end{array}$ \\
\hline $\begin{array}{l}\text { CASIA } \\
\text { V4- Iris } \\
\text { Interval }\end{array}$ & 200 & 198 & 200 & 99 & 100 \\
\hline $\begin{array}{c}\text { CASIA } \\
\text { V4- Iris } \\
\text { Lamp }\end{array}$ & 200 & 192 & 194 & 96 & 97 \\
\hline $\begin{array}{c}\text { CASIA } \\
\text { V4- Iris } \\
\text { Syn }\end{array}$ & 200 & 193 & 197 & 96.5 & 98.5 \\
\hline $\begin{array}{l}\text { CASIA } \\
\text { V4- Iris } \\
\text { Thousand }\end{array}$ & 200 & 181 & 185 & 90.5 & 92.5 \\
\hline $\begin{array}{c}\text { CASIA } \\
\text { V4- Iris } \\
\text { Twins }\end{array}$ & 200 & 182 & 183 & 91 & 91.5 \\
\hline Total & 1000 & 946 & 959 & 94.6 & 95.9 \\
\hline
\end{tabular}

\section{CONCLUSIONS}

The segmentation results are mainly depends on how properly preprocessing of eye images are carried out. Preprocessing may affected by lightning environment, image capture 


\section{Asian Journal of Convergence in Technology ISSN NO: 2350-1146 I.F-5.11}

distance and other noise factors. Once preprocessing execute well then segmentation result will be much better. Overall classification results are depends on segmentation accuracy. As proper segmentation achieved then surely classification results will be proper. Hough transform play an important role for locating the region of interest i.e. iris. Gabor filter provide the feature that will encode by phase quantization method. Feature extraction and encoding is very crucial as classification is totally based on encoded features. The main contribution of this research work is hide in accuracy of classification results. To evaluate the classification result we implement RBF kernel and polynomial kernel along with fuzzy system. The classification accuracy given by RBF kernel is $95.9 \%$, polynomial kernel is $94.6 \%$ and by FCM $95.9 \%$. Based on these results we can conclude that RBF kernel is slightly more accurate in terms of classification accuracy.

\section{REFERENCES}

1 J. Daugman (2004). "How iris recognition works", IEEE Trans. CSVT, vol. 14 , no. 1 , pp. $21-30$.

2 J. Daugman (2003). "The importance of being random: statistical principles of iris recognition", Journal of pattern recognition society, page 279-291.

3 Chung-Chih Tsai, Heng-Yi Lin, Jinshiuh Taur ad Chin-Wang Tao (2012). "Iris recognition using possibilistic fuzzy matching on local feature". IEEE Transaction on systems, man and cybernatics.Vol.2.No.1

4 J.Daugman(2007).“New methods in iris recognition". IEEE transaction on systems, man and cybernatics. Vol.37.No.5.

$5 \quad$ K.Seetharaman and R. Raghupathy (2012). "Iris recognition for personal identification system". Procedia engineering (38) page 1531-1546.

6 J. G. Daugman (1994). "Biometric personal identification system based on iris analysis," U. S. Patent 5,291,560.

7 Wildes et al.(1996). "Automated, noninvasive iris recognition system and method".U.S. patent 5,572,596.

8 R.P. Wildes (1997). "Iris Recognition: An Emerging Biometric Technology", Proceedings of the IEEE, vol.85, pp.1348-1363

9 Kaushik Roy, Prabir Bhattacharya, Chin Y.Suen (2011). "Towards nonideal iris recognition based on level set method, genetic algorithms and adaptive asymmetrical SVMs". Engineering application of artificial intelligence (24) page no.458-574.

10 N. Ritter (1999). "Location of the Pupil-Iris Border in Slit Lamp Images of the Cornea", Proceedings of the International Conference on Image Analysis and Processing.

11 S. Lim, K. Lee, O. Byeon, and T.Kim (2001). "Efficient Iris Recognition through Improvement of Feature Vector and Classifier", ETRI Journal, vol. 23 , no.2, pp. 61-70.

12 Zhaofeng He, Zhenan Sun(2009). "Towards Accurateand Fast Iris Segmentation for Iris Biometrics "IEEE Transactions on Pattern Analysis and Machine Intelligence, Vol.31, No.9, September 2009

13 J. Cui, Y. Wang, T. Tan, L. Ma, and Z. Sun (2004). "A Fast and Robust Iris Localization Method Based on Texture Segmentation", SPIE Defense and Security Symposium, vol. 5404, pp. 401-408.

14 W. Kong and D. Zhang (2001). "Accurate iris segmentation based on novel reflection and eyelash detection model", Proceedings of 2001 International Symposium on Intelligent Multimedia, Video and Speech.

15 D. M. Monro, S. Rakshit, and D. Zhang (2007). "DCT Based Iris Recognition", IEEE Transactions on Pattern Analysis and Machine Intelligence, vol. 29, no. 4.

16 D. Field (1987). "Relations between the statistics of natural images and the response properties of cortical cells", Journal of the Optical Society of America.

\section{Volume V Issue I}

17 Ma L., Tan T., Wang Y., and Zhang D. (2003). Personal identification based on iris texture analysis. IEEE Trans. Pattern Anal. Mach. Intelligence, 25(12):1519 - 1533.

18 Makram Nabti, Lahouari Ghouti and Ahmed Bouridane," An effective and fast iris recognition system based on a combined multiscale feature extraction technique", Elsevier-Pattern Recognition 41, (2008) 868-879

19 C. Sanchez-Avila, R. Sanchez-Reillo, and D. De Martin- Roche (2002). "Iris-Based Biometric Recognition Using Dyadic Wavelet Transform", IEEE AESS System Magazines, vol. 17, no. 10, pp. 3-6.

20 Y. Zhu, T. Tan, and Y. Wang (2000). "Biometric Personal Identification Based on Iris Patterns", Proceedings of the 15th International Conference on Pattern Recognition, vol. 2, pp. 2801-2804.

21 Kaushik Roy, Prabir Bhattacharya and Ramesh Chandra Debnath (2007). "Multi-Class SVM Based Iris Recognition", international conference on computer and information technology.

22 L. Ma, Y. Wang, and T. Tan (2002), "Iris Recognition Based on Multichannel Gabor Filtering," Proc. Fifth Asian Conf. Computer Vision, vol. I, pp. 279-283,

23 A. Poursaberi and B.N. Araabi (2007). "Iris Recognition for Partially Occluded Images: Methodology and Sensitivity Analysis", EURASIP Journal on Advances in Signal Processing, vol 2007.

24 A. Poursaberi and B.N. Araabi (2005). "A Novel Iris Recognition System Using Morphological Edge Detector and Wavelet Phase Features", GVIP (05), No. V6, pp. 9-15.

25 W. Boles and B. Boashash (1998). "A human identification technique using images of the iris and wavelet transform", IEEE Transactions on Signal Processing, vol. 46, no. 4.

26 L. Ma, T. Tan, Y. Wang, and D. Zhang (2004). "Efficient Iris Recognition by Characterizing Key Local Variations", IEEE Trans. Image Processing, vol 13, no.6, pp. 739-750.

27 Birgale, L.V. and M. Kokare, (2009). "Iris recognition using discrete wavelet transform". Proceedings of the IEEE International Conference, Mar. 7-9,IEEE Xplore Press, Bangkok, pp: 147-151. DOI: 10.1109/ICDIP.2009.30

28 “A. Panganiban, N. Linsangan, F. Caluyo, (2012). Wavelet-based feature extraction algorithm for an iris recognition system".J.Inf.prpocess.Syst.,7(3) pp. 425-434.

29 C. Tisse, L. Martin, L. Torres, and M. Robert. (2002). "Person identification technique using human iris recognition", International Conference on Vision Interface.

30 G. Xu, Z. Zhang and Y. Ma (2008), "A novel method for iris feature extraction based on intersecting cortical model network", Journal of Applied Mathematics and Computing, 26, pp. 1-2.

31 H. Rai and A. Yadav, "Iris Recognition using Combined Support Vector Machine and Hamming Distance Approach", Expert System with Applications, Vol. 41, pp 588- 593, 2014.

32 K. Roy and P. Bhattacharya. Optimal features subset selection and classification for iris recognition. J. Image Video Process., 2008:9:19:20, 2008.

33 Nor'aini et.al. (2013). "Classification of iris regions using principal component analysis and support vector machine". IEEE International Conference on Signal and Image Processing Applications (ICSIPA) .

34 H. A. Park and K. R. Park, Iris recognition based on score level fusion by using SVM, Pattern Recognition Letters, vol.28, no.15, pp.20192028, 2007.

35 Usham Dias, Vinita Frietas, Sandeep P.S and Amanda Fernandes, "A Neural Network Based Iris Recognition System for Personal Identification", ICTACT Journal On Soft Computing, Vol. 1, No. 2, pp. 78-84, 2010.

36 Mrunal M. Khedkar, S. A. Ladhake (2013). "Neural network based human iris pattern recognition system using SVD transform features".ISSN vol.1page no.2320-8945.

37 Broussard, R., Kennell, L., Ives, R., \& Rakvic, R. (2008). An artificial neural network based matching metric for iris identification. In J. Astola, 
ISSN NO: 2350-1146 I.F-5.11

K. Egiazarian, \& E. Dougherty (Eds.), Image processing: Algorithms and systems VI. Proceedings of SPIE (Vol. 6812, pp. 68120S-68120S11).

38 Fadi N. Sibai.Hafsa I. Hosani, raja M. Naqbi, Salima Dhanhani, Shaikha Shehhi, "Iris Recognition Using Artificial Neural Networks", Elsevier, Expert Systems with Applications, Vol. 38, No. 5, pp. 5940-5946, 2011.
39 Thiyam Meetei, Shahin Begum (2014). "A comparative study of feature extraction and classification methods for iris recognition", International journal of computer application, vol 2. 
